\title{
Thermal membrane potential and thermoosmosis across charged membranes
}

\author{
Masayasu Tasaka \\ Department of Industrial Chemistry, Faculty of Engineering, Shinshu \\ University, Wakasato, Nagano 380 , Japan
}

\begin{abstract}
When different temperatures are given to simple electrolyte solutions on both side of a membrane, there appears an electrostatic potential, and osmotic flow of water occurs through the membrane. Thermal membrane potential was determined by flushing two solutions having different temperatures on the two surfaces of a thick membrane to remove diffusion layers. Thermoosmotic flow was observed using a capillary. The effective temperature difference working for thermoosmosis was estimated by measuring the thermal membrane potential across the membrane. Experimental data thus obtained were compared with a theory based on nonequilibrium thermodynamics.
\end{abstract}

\section{INTRODUCTION}

When two solutions of a simple electrolyte are placed on both sides of a membrane and no electric current is passing through the membrane, various phenomena such as membrane potential, solute flux, solvent flux, etc. are simultaneously found. The driving force for those phenomena is also various: concentration difference, pressure difference, temperature difference, etc. Although individual phenomenon has been studied in detail, it is most important to study all these related phenomena on the basis of a common theory. We have been working to understand various membrane phenomena observed using a simple membrane when there is no electric current and the hydrostatic pressures are equal, consistently based on a nonequilibrium thermodynamics (refs. 1-15). In this paper, our works on nonisothermal membrane phenomena are summerized(refs. 1-9).

The membranes used in the present work are mainly sulfonated polystyrene-collodion interpolymer membranes, which can be regarded as a kind of polyelectrolyte solution. A charge-mosaic membrane made of pentablock copolymer, which is composed of cation- and anion-exchange elements and, consequently, has very high permeability for simple electrolytes, is also used for studying the interaction between solute flux and water flux(ref. 16). The electrolyte solutions placed on both sides of those membranes are generally $1-1$ electrolytes.

In literature including our own works, thermal membrane potential is always found to be positive at the hot side for cation permselective membranes and negative for anion permselective membranes, respectively.

Concerning the direction of thermoosmosis, however, the conclusions reported in literature are contradictory. In case of charged membranes the direction of thermoosmosis through membrane is mostly from the cold side to the hot side (refs. $2,5,9,17,18$ ). For noncharged and/or low charged membranes, however, the direction of thermoosmosis in water or aqueous solutions is from the hot side to the cold side (refs. 17,19-23). Moreover, it was observed that the direction of thermoosmosis also changes with change in temperature of external solutions (refs. 24,25 ), pore size of membranes (refs. 19,26), and solvent (ref. 21). The magnitude of the thermoosmotic coefficient changes with the change in the mean temperature of both external solutions (refs. $17,20,22,23,25,27,28)$, and also with the salt concentration(refs. 5,9,21, $27,28)$.

In closed salt solution systems having no membrane, solvent (water) moves toward the hot side and solute (potassium chloride, sodium chloride, etc.) towards the cold side under a temperature gradient (ref. 29).

The permeation of electrolyte through membranes due to temperature difference was so small that the change of salt concentration could not be found when dealing with charged membranes. When the membrane : used was a charge-mosaic membrane, electrolytes could pass through the membrane so easily that the direction of electrolyte was found to be from the cold side to the hot side, in agreement with thermal membrane potential. 


\section{BASIC EQUATION OF MEMBRANE PHENOMENA}

Applying nonequilibrium thermodynamics to ion transport processes with no chemical reaction, in mechanical equilibrium, the entropy production $\sigma$ may be given by (refs. 30,31 )

$$
-T \sigma=J_{s} \cdot \operatorname{grad} T+\sum_{i} J_{i} \cdot \operatorname{grad} \tilde{\mu}_{i}
$$

where

$$
\tilde{\mu}_{i}=\mu_{i}+v_{i} P+z_{i} F \psi
$$

$J_{S}$ is the total entropy flux and $J_{i}$ is the absolute mass flux. In addition $\tilde{\mu}_{i}$ is the chemical potential including contributions due to external forces, $\mu_{i}$ the chemical potential depending on temperature and composition, $v_{i}$ the partial molar volume, $z_{i}$ the charge number of component $i, P$ the pressure, $F$ the Faraday constant, $T$ the absolute temperature, and $\psi$ is the electrostatic potential. Thus, the phenomenological equations for the fluxes relative to membrane are given by

$$
\begin{aligned}
& -J_{s}=\bar{L}_{s s} \operatorname{grad} \bar{T}+\sum_{i} \bar{L}_{s i} \operatorname{grad} \overline{\tilde{\mu}}_{i} \\
& -J_{i}=\bar{L}_{i s} \operatorname{grad} \bar{T}+\sum_{j} \bar{L}_{i j} \operatorname{grad} \overline{\tilde{\mu}}_{j}
\end{aligned}
$$

where the parameters in the membrane phase are denoted by a overbar.

Let us assume that all gradients in the membrane are in the $x$ direction perpendicular to the membrane surfaces and, at steady state, temperature $T$, and general chemical potential, $\tilde{\mu}_{i}$, at points $x=0$ and $x=\delta$ in the membrane (at opposite surfaces of the membrane), are equal to those in the contiguous solutions. That is,

$$
\begin{aligned}
& \Delta \tilde{\mu}_{i}=\tilde{\mu}_{i}(\delta)-\tilde{\mu}_{i}(0)=\overline{\tilde{\mu}}_{i}(\delta)-\overline{\tilde{\mu}}_{i}(0)=\Delta \overline{\tilde{\mu}}_{i} \\
& \Delta T=T(\delta)-T(0)=\bar{T}(\delta)-\bar{T}(0)=\Delta \bar{T}
\end{aligned}
$$

where $\Delta$ shows the difference between two fluid phases on either side of the membrane. By solving eqs. (3) and (4) for forces and integrating them from one side of the membrane to the other, keeping the fluxes constant, we have(refs. $2,8,32,33$ )

$$
\begin{aligned}
& -J_{s}=L_{s s} \Delta T+\sum_{i} L_{s i} \Delta \tilde{\mu}_{i} \\
& -J_{i}=L_{i s} \Delta T+\sum_{j} L_{i j} \Delta \tilde{\mu}_{j}
\end{aligned}
$$

where $L_{\alpha \beta}$ is the permeability coefficient of the membrane of finite thickness.

If $\Delta T=0$, from eq. (8) we have

$$
-J_{i}=\sum_{j} L_{i j} \Delta \tilde{\mu}_{j} \quad \text { or } \quad-\Delta \tilde{\mu}_{j}=\sum_{i} R_{j i}{ }_{i}
$$

For solvent flux, $J_{0}$, that is the osmotic flow through the membrane, we have (ref. 6)

$$
-J_{0}=\left(1 / R_{00}\right) \Delta \mu_{0}+\sum_{\mathrm{i}}\left(R_{0 \mathrm{i}} / R_{00}\right) J_{\mathrm{i}}
$$

and electric current, $I$, can be written as

$$
I=\sum_{i} z_{i}^{F J}
$$

The physical quantities which characterized the membrane properties are also written as

$$
\begin{aligned}
& t_{i}=\left(z_{i} F J_{i} / I\right) \Delta T=0, \Delta P=0, \Delta \mu_{i}=0 \\
& \tau_{i}=\left(J_{i} / I\right) \Delta T=0, \Delta P=0, \Delta \mu_{i}=0 \\
& \tau_{0}=\left(J_{0} / I\right) \Delta T=0, \Delta P=0, \Delta \mu_{0}=0=-\left(1 / R_{00}\right) \sum_{i}^{\prime}\left(R_{0 i} t_{i} / z_{i} F\right)
\end{aligned}
$$

where the $\operatorname{sum} \sum^{\prime}$ is taken over all ionic species without water.

If the membrane is ideally permselective for component $i, i . e ., t_{i}=1$, the value of $R_{0 i} / R_{00}$ ' which means the interaction between water and component $i$, can be directly obtained by measuring electroosmosis. Here, we would like to point out that the interactions between water and salt fluxes, $R_{0 \mathrm{i}} / R_{00}$, are most important to understand the osmotic flow of water.

The membrane potential can be calculated from the condition $I=0$ and $\Delta P=0$ (ref. 1$)$. That is, 


$$
-\Delta \psi=n \Delta T+\sum_{i} \tau_{i} \Delta \mu_{i}
$$

where the thermoelectric potential coefficient is

$$
n=\sum_{j}{ }_{j} L_{j s} / \sum_{i} \sum_{j} z_{i} z_{j} L_{i j} F
$$

and the reduced transport number of species $i$ is

$$
\tau_{i}=\sum_{j}{ }_{j} L_{j i} / \sum_{i} \sum_{j}{ }_{i}{ }^{z}{ }_{j}{ }_{i j} F
$$

Equation (17) is derived from eq. (13) using the Onsager reciprocal relationship $L_{i j}=L_{j i}$. Moreover, if the transported entropy $\overline{\bar{s}}_{j}$ is defined in terms of the phenomenological coefficients(refs. 32-34)

$$
L_{i s}=\sum_{j} L_{i j} \overline{\bar{s}}_{j}
$$

from eq. (16) we have (ref. 6)

$$
\eta=\sum_{j} \tau_{j} \overline{\bar{s}}_{j}
$$

$\Delta \mu_{i}$ in eq. (15) is composed of two terms due to the temperature difference and due to the composition:

$$
\Delta \mu_{i}=-s_{i} \Delta T+\left(\Delta \mu_{i}\right)_{T}
$$

where

$$
s_{i}=s_{i}^{\circ}-R \ln a_{i}-R T\left(\partial \ln a_{i} / \partial T\right)
$$

$s_{i}$ is the partial molar entropy of component $i$.

Substituting eqs. (19) and (20) into eq. (15), we have(ref. 6)

$$
-\Delta \psi=\sum_{i} \tau_{i}\left\{\left(\overline{\bar{s}}_{i}-s_{i}\right) \Delta T+\left(\Delta \mu_{i}\right)_{T}\right\}
$$

Similarly, for the mass flux of component $i$ we have

$$
-J_{i}=\sum_{j}\left(L_{i j}-\tau_{i} \tau_{j} F^{2} L_{e}\right)\left\{\left(\overline{\bar{s}}_{j}-s_{j}\right) \Delta T+\left(\Delta \mu_{j}\right)_{T}\right\}
$$

where $L_{e}$ is the electric permeability (refs. 35,36$)$ :

$$
L_{e}=\sum_{i} \sum_{j} z_{i} z_{j} L_{i j}
$$

In the case of $\Delta T=0$ we have the well-known equation for concentration membrane potential,

$$
-\Delta \psi=\sum_{i} \tau_{i}\left(\Delta \mu_{i}\right)_{T}
$$

The permeation velocity of salt is given by eq. (9) and from eq. (23) the water flux is given by

$$
-J_{0}=\sum_{j}\left(L_{0 j}-\tau_{0} \tau_{j} F^{2} L_{e}\right)\left(\Delta \mu_{j}\right)_{T}
$$

which is equivalent to eq. (10). $\Delta \psi$ and $J_{S}$ are not discussed in this paper but a brief discussion will be given to $J_{0}$ at $\Delta T=0$ in relation to the nonisothermal osmosis.

In nonisothermal systems and also if there is no concentration difference on both sides of membrane, the volume flux, $J_{v}=\sum_{i} v_{i} J_{i}$, due to $\Delta T$ (thermoosmosis) may be calculated from eq. (23) as (ref. 8)

$$
-J_{\mathrm{v}}=D \Delta T
$$

where

$$
D=\sum_{i} v_{i} \sum_{j}\left(L_{i j}-\tau_{i} \tau_{j} F^{2} L_{e}\right)\left(\overline{\bar{s}}_{j}-s_{j}\right)
$$

The above equations can be applied to every systems, but in the following the application of the equations is limited to $1-1$ electrolyte systems with cation permselective membranes for simplicity, except specified cases. 


\section{INTERACTION BETWEEN IONS AND WATER}

Volume flow is observed through a membrane if two solutions of different concentrations of an electrolyte are separated by the membrane. However, the volume flow is not always proportional to the difference in the osmotic pressure on the two sides of the membrane if the membrane has fixed charges. Such anomalous membrane phenomena are caused by a strong interaction between the flows of ions and water. The reduced transport number of water is a measure of the interaction. From eq. (15) the reduced transport number of water can be written as

$$
\tau_{0}=-\left(R_{0+} / R_{00}\right) t_{+}+\left(R_{0-} / R_{00}\right) t_{-}
$$

As seen from eq. (10) $R_{0+} / R_{00}$ means the interaction between cation and water or the number of moles of water accompanied with one mole of transported ions. If the membrane is ideally permselective for counterions, we can obtain the value of $R_{0+} / R_{00}$ or $R_{0-} / R_{00}$ from the electroosmotic experiments. These values are approximately inversely proportional to the mobilities of these ions (ref. 13). In the case $I=0$, from eq. (10) the solvent flux can be written as

$$
-J_{0}=\left(1 / R_{00}\right) \Delta \mu_{0}+\left(R_{0+} / R_{00}+R_{0-} / R_{00}\right) J_{ \pm}
$$

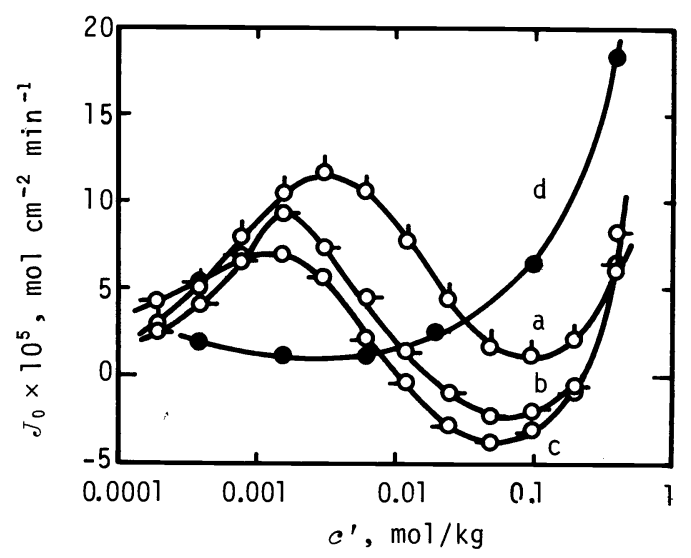

Fig. 1. Examples of solvent flow through membranes in solution of various electrolytes: $c^{\prime \prime} / c^{\prime}=8 ; \mathrm{i}-\mathrm{m}: \mathrm{a}, \mathrm{KCl} ; \mathrm{b}, \mathrm{NaCl} ; \mathrm{c}$, LiCl; collodion membrane: d, $\mathrm{KCl}$ (ref. 10). Experiments at $c^{\prime \prime} / c^{\prime}=2$ and 4 are similar.

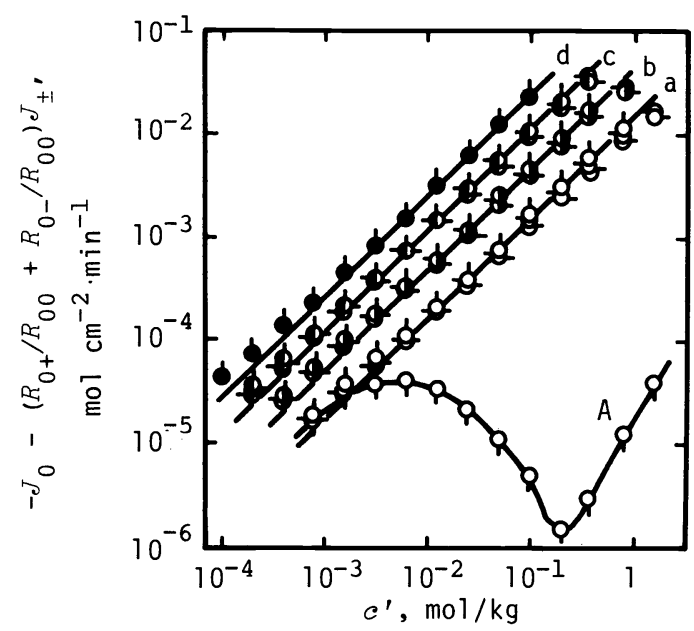

Fig. 2. The dependence of $-J_{0}-\left(R_{0+} / R_{00}+\right.$ $\left.R_{0-} / R_{00}\right) J_{ \pm}$on molality of various electrolytes for membrane i-m(ref. 10). $c^{\prime \prime} / c^{\prime}$ : $a, 2 ; b, 4 ; c, 8 ; d, 16$. Electrolytes: $b$, $\mathrm{KCl} ; 0, \mathrm{NaCl} ;-\infty, \mathrm{LiCl} . \mathrm{A}$, the dependence of $J_{0}$ on molality of $\mathrm{KCl}$ at $c^{\prime \prime} / c^{\prime}=2$. where $J_{ \pm}$is the salt flux. Since $\Delta \mu_{0}$ is proportional to the concentration difference, $c$ " $-c^{\prime}, \log \left[-J_{0}-\left(R_{0+} / R_{00}+R_{0-} / R_{00}\right) J_{ \pm}\right]$must be linear to $\log C^{\prime}$ with the slope of unity.

Figure 1 shows examples of the solvent flux through sulfonated polystyrene-collodion interpolymer membrane $i-m$ in solution of various electrolytes where the concentration ratio of two solutions $c^{\prime \prime} / c^{\prime}=8$. In solutions of sodium chloride and lithium chloride, the socalled negative osmosis was observed because the second term of eq. (30) becomes larger than the first term. Figure 2 shows the relationship between the calculted values of log $\left[-J_{0}-\left(R_{0+} / R_{00}+R_{0-} / R_{00}\right) J_{+}\right]$and the electrolyte concentration. All the plots are found to be linear with a slope of approximately unity, as expected from the theory.

Similarly, the water flux through chargemosaic membranes are shown in Fig. 3. In the range 0.001 to $0.1 \mathrm{~mol} / \mathrm{dm}^{3}$, negative osmosis is shown for potassium chloride, sodium chloride, and lithium chloride solutions because of the high salt fluxes(ref. 14).

Thus, the interaction between ions and water plays an important role on solvent flux or volume flux across membranes. Anomalous

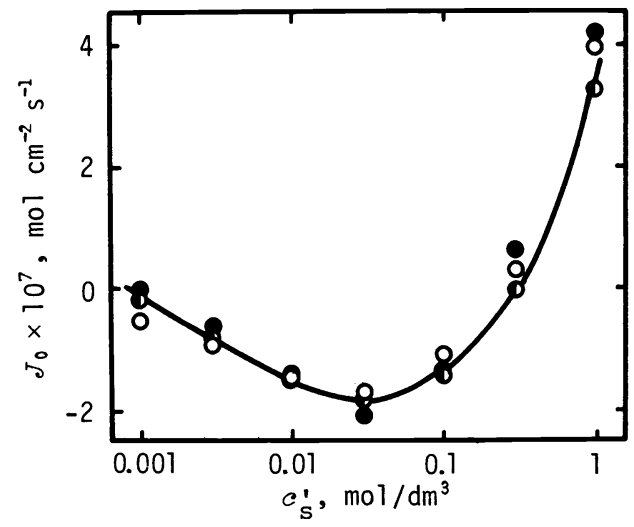

Fig. 3. Water fluxes, $J_{0}$, through chargemosaic membrane TUN-1002-QS against lower electrolyte concentration, $c_{S}^{\prime}$. Electrolytes: $0, \mathrm{KCl} ; \bullet, \mathrm{NaCl} ; 0, \mathrm{LiCl} . c_{\mathrm{S}}^{\prime \prime} / c_{\mathrm{S}}^{\prime}$ $=2$ (ref. 14). 
osmosis and negative osmosis through low charged membranes and charge-mosaic membranes can well be explained by eq. (30) (refs. 10-12,37,38).

\section{THERMAL MEMBRANE POTENTIAL}

If there is no difference in molalities of electrolyte across the membrane, from eq. (15) $-\Delta \psi / \Delta T$ is given by (refs. $1,38,39)$

$$
\begin{aligned}
-\Delta \psi / \Delta T & =n-\sum_{\mathbf{i}} \tau_{i}\left\{s_{i}^{\circ}-R \ln \alpha_{i}-R T\left(\partial \ln a_{i} / \partial T\right)\right\} \\
& \approx n-\sum_{\mathbf{i}} \tau_{\mathbf{i}}\left(s_{\mathbf{i}}^{\circ}-R \ln \alpha_{i}\right)
\end{aligned}
$$

If the membrane is ideally permselective for counterions, i.e., $t_{+}=1$, from eq. (31) we have

$$
-\Delta \psi / \Delta T=(R / F) \ln \alpha_{+}+\alpha_{+}
$$

for cation-exchange membranes, where

$$
\alpha_{+}=\eta-s_{+}^{\circ} / F-\tau_{0} s_{0}
$$

Membrane potential in nonisothermal systems was measured by flushing the two solutions having different temperatures on the two surfaces of a thick membrane to remove diffusion layers(ref. 1). Membrane potential cell is schematically shown in Fig. 4. The nozzle of the inlet tube, whose internal diameter was 2 to $3 \mathrm{~mm}$, was fixed at a position 2 to $3 \mathrm{~mm}$ apart from the membrane surface. While in isothermal systems a reproducible stationary membrane potential was observed at relatively low flow rates of electrolyte solutions $(60-150$ $\left.\mathrm{cm}^{3} / \mathrm{min}\right)$ and with thin membranes, in nonisothermal systems high flow rates $\left(250-300 \mathrm{~cm}^{3} / \mathrm{min}\right)$ and thicker membranes (of about $1 \mathrm{~mm}$ thickness) were required to obtain reproducible membrane potentials independent of the flow rate. An example of the dependence of membrane potential on flow rate is shown in Fig. 5 .

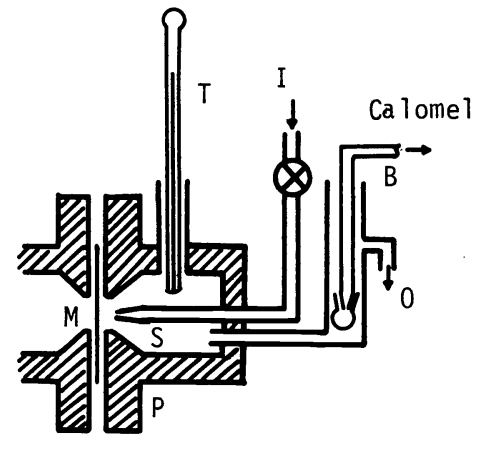

Fig. 4. Membrane potential cell(ref. 1). $B$, salt bridge; $I$, solution inlet; $M$, membrane; $O$, solution outlet; $S$, solution; $T$, thermometer.

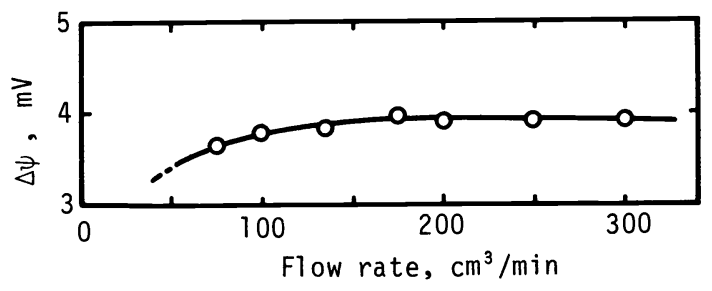

Fig. 5. The influence of flow rate on membrane potential for heterogeneous cationexchange membrane $\mathrm{M}-10 / 0$ at $0.1 \mathrm{~mol} / \mathrm{kg} \mathrm{KCl}$ solution(ref. 1). Membrane thickness, 1.13 $\mathrm{mm} ; \Delta T, 10 \mathrm{~K}$.

In the range of small temperature differences $(<10 \mathrm{~K})$, linear relationships were observed between $\Delta \psi$ and the temperature difference, $\Delta T$ (refs. 1-3). The electrical potential of the hot side solution was always positive for cation permselective membranes and negative for anion permselective membranes. These phenomena mean that ions are forced to move from the hot side to the cold side across a membrane.

In Figs. 6 and 7 , the temperature coefficient of the thermal membrane potential, $-\Delta \psi / \Delta T$, with cation-exchange membranes is plotted as a function of activities of electrolytes(refs. $3,40)$. The absolute value of $-\Delta \psi / \Delta T$ decreases with increasing ionic radius of hydrated alkali metal ions. As shown by eq. (33), $\alpha_{+}$is made of three terms: $\eta_{,} s_{+}^{\circ} / F_{\text {, }}$ and $\tau_{0} s_{0}$. The value of $s_{+}$are known as the molar entropy of ions referred to that of hydrogen ion and the value of $s_{0}$ may be approximated by the molar entropy of water, i.e., $s_{0}=3.99 \mathrm{~J} \mathrm{~K}^{-1}$ $\mathrm{mol}^{-1}$, neglecting the effect of hydration of ions on the molar entropy of water (ref. 41). The value of $\tau_{0}$ can be obtained by the measurement of electroosmosis. Thus, using eq. (33) we can calculate values of pure thermoelectric coefficient $n$. These values are also shown in Tables 1 and 2. The value of $\eta$ increases with increasing the mobility of counterions. In general, the absolute value of $-\Delta \psi / \Delta T$ becomes high when the water content of the membrane is low, because $\tau_{0}$ is small and the value of the transported entropy of counterions $\overline{\bar{s}}_{+}$would be much smaller. For anion-exchange membranes(ref. 3), phospholipid bilayer films (refs. 40, 
42), and anion permselective liquid membranes (refs, 43-45), the theory agreed with experimental results.

For polyvalent type electrolyte solutions we have

$$
-\Delta \psi / \Delta T=\left(R / z_{+} F\right) \ln \alpha_{+}+\alpha_{+}
$$

for cation-exchange membranes, where

$$
\alpha_{+}=n-s_{+}^{0} / z_{+} F-\tau_{0} s_{0}=\left(\overline{\bar{s}}_{+}-s_{+}^{0}\right) / z_{+} F+\tau_{0}\left(\overline{\bar{s}}_{0}-s_{0}\right)
$$

The slopes of the temperature coefficient of thermal membrane potential, $-\Delta \psi / \Delta T$, against logarithmic activities of counterions in the external solution were predicted to be $R / 2 F$ for 2-1 electrolytes with cation-exchange membranes and $-R / 2 F$ for $1-2$ electrolytes with anionexchange membranes, respectively (ref. 6).

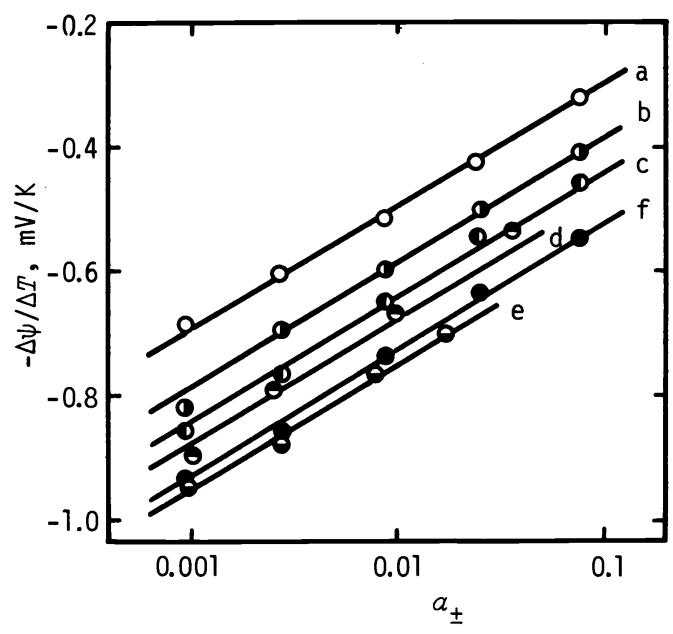

Fig. 6. The effect of counterions on the temperature coefficient of the membrane potential, $-\Delta \psi / \Delta T$, for cation-exchange membrane M-10/0 (ref. 3). Electrolytes: a, Li $\mathrm{Cl} ; \mathrm{b}, \mathrm{NaCl} ; \mathrm{c}, \mathrm{KCl} ; \mathrm{d}, \mathrm{RbCl} ; \mathrm{e}, \mathrm{CsCl} ; \mathrm{NH}_{4}$ $\mathrm{Cl}$.

If the membrane is not ideally permselective, the transport number $t_{i}$ lower than unity must be taken into account. If experimental value of $t_{i}$ is introduced into eq. (31), the experimental data for thermal membrane potential can be explained. In particular, if a membrane is a charge-mosaic membrane which is composed of ideal cation- and anion-exchange elements, $n$ can be written as the sum of the combination from two parts:

$$
\begin{aligned}
n & =t_{+} n^{\mathrm{c}}+t_{-} \eta^{\mathrm{a}} \\
& =\left(t_{+} \overline{\bar{s}}_{+}^{\mathrm{c}}-t_{-} \overline{\bar{s}}_{-}^{\mathrm{a}}\right) / F+\tau_{0}^{\mathrm{c}} \bar{s}_{0}^{\mathrm{c}}+\tau_{0}^{\mathrm{a}=\mathrm{a}}{ }_{0}
\end{aligned}
$$

where the superscripts $c$ and a refer to a cation permselective element and an anion permselective element, respectively. Using the overall transport number, $t_{i}$, we have (ref. 7)

$$
\begin{aligned}
-\Delta \psi / \Delta T= & \left(2 t_{+}-1\right)(R / F) \ln a_{ \pm} \\
& +t_{+} \alpha_{+}^{c}+t_{-} \alpha_{-}^{a}
\end{aligned}
$$

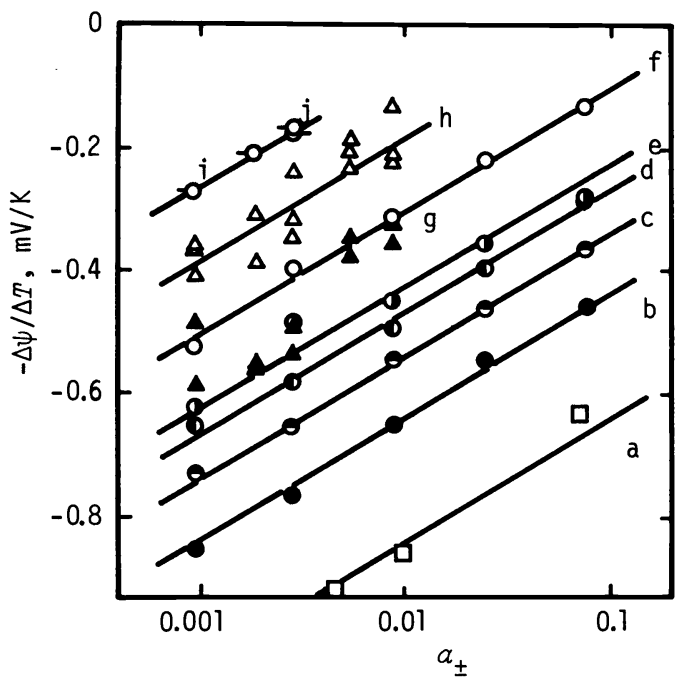

Fig. 7. Temperature coefficients of the membrane potential, $-\Delta \psi / \Delta T$, for various cationexchange membranes: Notations are the same shown in Table 2 (refs. $3,5,40)$. a, in $\mathrm{NaCl}$ solutions

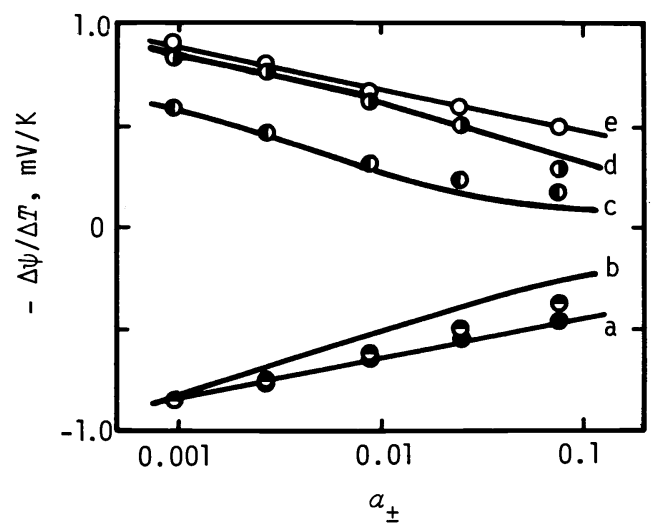

Fig. 8. The dependence of temperature coefficient, $-\Delta \psi / \Delta T$, on $\mathrm{KCl}$ molality (ref. 7). Membranes: $a, M-10 / 0 ; b, M-8 / 2 ; c, M-4 / 6 ; d$, $\mathrm{M}-3 / 7 ; \mathrm{e}, \mathrm{M}-0 / 10$. 
Heterogeneous cation- and anion-exchange membranes(M-10/0, M-0/10) and three heterogeneous mixed ion-exchange membranes $(M-8 / 2, M-4 / 6, M-3 / 7)$ were prepared from $60 \%$ ion-exchange resin (cation-exchange resin: Amberlite XE-69 and anion-exchange resin: Amberlite XE-119 of about 300 mesh) and 408 poly (vinyl chloride) as a binder. The values of $\alpha q$ and $\alpha$ a can be obtained from the experimental data of thermal membrane potential with ideally permselective membranes $M-10 / 0$ and $M-0 / 10$. If the overall transport numbers of cations and anions in membranes are obtained, the values of $-\Delta \psi / \Delta T$ for membranes $M-8 / 2, M-4 / 6$, and $M-3 / 7$ can be calculated from eq. (37). In Fig. 8 the experimental data of thermal membrane potential are compared with the calculated values shown by the solid lines. Considering the experimental error, the agreement is good (ref. 7).

TABLE 1. The values of $\alpha_{+}, \tau_{0}$, and $n$ with membrane $M-10 / 0$ (ref. 3 )

\begin{tabular}{llll}
\hline Counterion & $\tau_{0} F$ & $\alpha_{+^{\prime}} \mathrm{mV} / \mathrm{K}$ & $\eta_{,} \mathrm{mV} / \mathrm{K}$ \\
\hline $\mathrm{Li}^{+}$ & 6.64 & -0.10 & 4.48 \\
$\mathrm{Na}^{+}$ & 6.27 & -0.20 & 4.94 \\
$\mathrm{~K}^{+}$ & 6.05 & -0.24 & 5.20 \\
$\mathrm{Rb}^{+}$ & 5.91 & -0.29 & 5.24 \\
$\mathrm{Cs}^{+}$ & 6.04 & -0.36 & 5.40 \\
$\mathrm{NH}_{4}^{+}$ & 5.88 & -0.33 & 5.11 \\
\hline
\end{tabular}

TABLE 2. The properties of charged membranes in $\mathrm{KCl}$ solutions(refs $, 3,5,40$ )

\begin{tabular}{|c|c|c|c|c|c|c|}
\hline & Membrane & $\begin{array}{l}\text { Ion-exchange } \\
\text { capacitya }\end{array}$ & $\begin{array}{l}\text { Water } \\
\text { content b }\end{array}$ & ${ }^{\tau} 0^{F^{c}}$ & $\underset{\mathrm{mV} / \mathrm{K}}{\alpha_{+}}$ & $\stackrel{n}{\mathrm{mV} / \mathrm{K}}$ \\
\hline a & Bilayer: egg yolk phosphatidylcholine ${ }^{d}$ & - & - & - & -0.45 & - \\
\hline b & $\begin{array}{l}\text { Heterogeneous: sulfonated polystyrene } \\
+\operatorname{PVC}(408), M-10 / 0\end{array}$ & 1.89 & 0.37 & 6.05 & -0.24 & 5.20 \\
\hline c & Homogeneous: sulfonated polystyrene & 1.03 & 0.59 & 6.63 & -0.14 & 5.72 \\
\hline $\mathrm{d}$ & $\begin{array}{l}\text { Homogeneous: sulfonated polystyrene } \\
\text { (Nepton CR-61) }\end{array}$ & 1.21 & 0.43 & 22.6 & -0.07 & 17.36 \\
\hline e & $\begin{array}{l}\text { Homogeneous: sulfonated phenol resin } \\
\text { (Nepton CR-51) }\end{array}$ & 0.95 & 0.43 & 9.61 & -0.03 & 7.72 \\
\hline f & $\begin{array}{l}\text { Interpolymer: sulfonated polystyrene } \\
+ \text { dried collodion }(57 \%)\end{array}$ & 1.05 & 0.81 & 60 & 0.10 & 44.6 \\
\hline g & Liquid: sulfonated polystyrene $(14.28)$ & 3.80 & 6.0 & 150 & 0.05 & 109.7 \\
\hline $\mathrm{h}$ & Liquid: sulfonated polystyrene $(4.4 \%)$ & 3.80 & 21.7 & - & 0.21 & - \\
\hline$i$ & Oxidized collodion & - & 3.3 & - & 0.26 & - \\
\hline j & $\begin{array}{l}\text { Interpolymer: sulfonated polystyrene } \\
+ \text { dried collodion }(958)\end{array}$ & 0.25 & 2.0 & $550 e$ & 0.26 & 400 \\
\hline
\end{tabular}

a The units are millimole per gram of dry membrane. b Measured as grams of water per gram of $\mathrm{dry}$ membrane. $\mathrm{c}$ In $0.01 \mathrm{M}-\mathrm{KCl}$. $\mathrm{d}$ Measured in $\mathrm{NaCl}$ solutions. e Measured in $0.001 \mathrm{M}-\mathrm{KCl}$.

\section{THERMOOSMOSIS}

Although the bulk solutions on the two sides of the membrane were fairly strongly stirred in thermoosmotic experiments as shown in Fig. 9, it is nearly impossible to remove the stagnant layers with respect to temperature on the membrane surfaces.

To study the thermoosmosis quantitatively, therefore, it is important to know the temperature difference working effectively on both sides of membrane, $\Delta T$, which was always found to be different from the temperature difference in bulk solutions, $\Delta T_{b}$. Since the relationship between thermal membrane potential and $\Delta T$ is already established, $\Delta T$ may be estimated by measuring thermal membrane potential. However, the thermal membrane potential in the thermoosmotic cell cannot be measured while thermoosmosis is being observed. Therefore, the relationship between the temperature difference of bulk solutions, $\Delta T_{b}$, and the thermal membrane potential is first observed under the same experimental conditions as when thermoosmosis was measured. Since the relationship between $\Delta T$ and thermal membrane potential is already known, we can determine the relationship between $\Delta T$ and $\Delta T_{\mathrm{b}}$ at the experimental conditions for thermoosmosis. An example of the relationship between $\Delta T$ and $\Delta T_{\mathrm{b}}$ for sulfonated polystyrene -collodion interpolymer membrane $i-m-2$ is shown in Fig. 10. The value of $\Delta T / \Delta T_{b}=0.7$ (ref. 6).

Figure 11 shows an example of $J_{\mathrm{V}}$ against $\Delta T$ plot. From the slope, we can determine the thermoosmotic coefficient, $D$. For membrane $i-m-2$, thermoosmotic coefficient is plotted against potassium chloride molality in Fig.12. The staircase-type behavior of the electrolyte 


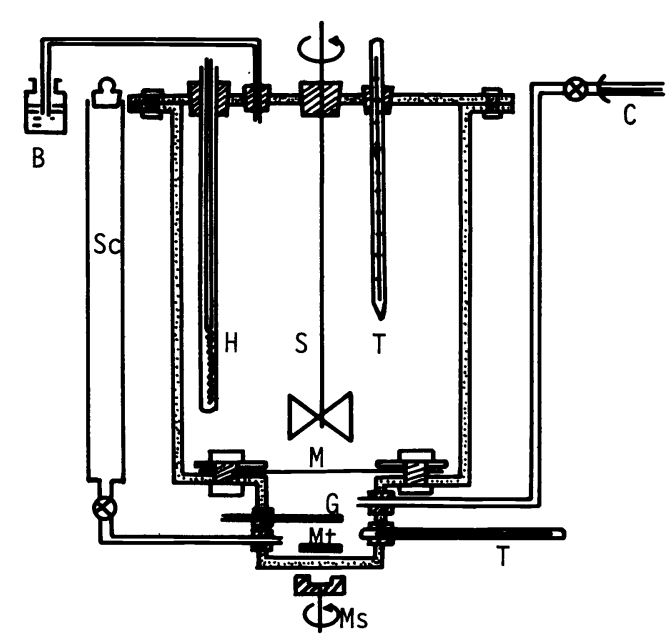

Fig. 9. The apparatus for measurement of thermoosmosis (ref. 5). B, bottle to adjust the solution level; C, capillary tube; G, glass tube for circulating cold water; $\mathrm{H}$, heater; M, membrane; Ms, magnetic stirrer; Mt, magnetic stirrer tip; S, stirrer; Sc, solution container; $T$, thermometer.

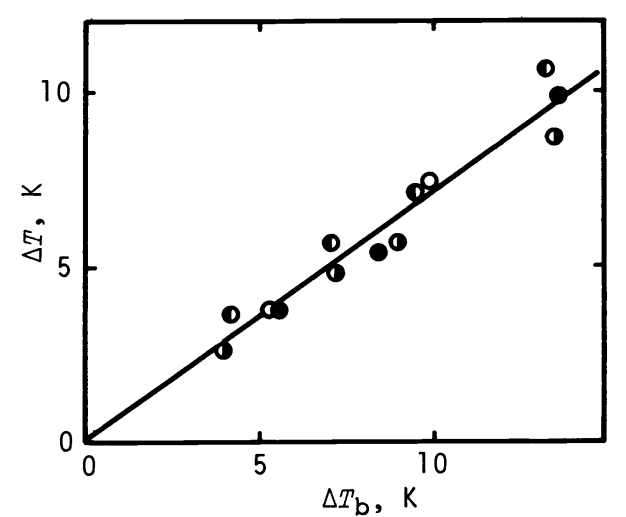

Fig. 10. The relationship between the effective temperature difference, $\Delta T$, and the temperature difference of two bulk solutions, $\Delta T_{\mathrm{b}}$ (ref. 5). $\mathrm{KCl}$ molalities $(\mathrm{mol} / \mathrm{kg})$ : $0,0.003 ; 0,0.01 ; 0,0.03 ; \bullet, 0.1$.

concentration dependence of $D$ in the figure may be commonly found for charged membranes(refs. $2,5,8,9)$.

If ions move from the hot side to the cold side across a membrane, the electrical potential of the hot side solution must be negative for cation permselective membranes. However, as mentioned above, it was observed that the electrical potential of the hot side solution was almost positive for cation permselective membranes and negative for anion permselective membranes. This means that the solute is forced to move toward the hot side.

If $\Delta \mu_{i}=0$, from eq. (28) we have (refs. 8,9 )

$$
D=D^{\circ}+D^{\prime}+D^{\prime \prime}
$$

where

$$
\begin{aligned}
& D^{\circ}=\bar{c}_{0} Z_{\mathrm{v} 0}\left(\overline{\bar{s}}_{0}-s_{0}\right) / \delta \\
& D^{\prime}=-\bar{c}_{0} F \phi X Z_{\mathrm{v} \pm}\left(\overline{\bar{s}}_{0}-s_{0}\right) / \delta \\
& D^{\prime \prime}=\left(\bar{c}_{-}+t_{-} \phi X\right) Z_{\mathrm{v} \pm}\left(\overline{\bar{s}}_{\mathrm{s}}-s_{\mathrm{s}}\right) / \delta
\end{aligned}
$$

and

$$
\begin{aligned}
& L_{i j}=\bar{c}_{j} z_{i j} / \delta \\
& \tau_{v i}=\sum_{j} v_{j} z_{j i} \\
& \bar{s}_{s}=\bar{s}_{+}+\overline{\bar{s}}_{-} \\
& \bar{c}_{+}=\bar{c}_{-}+\phi X
\end{aligned}
$$

We may assume that $\tau_{\mathrm{v}+}=\tau_{\mathrm{v}-}=\tau_{\mathrm{v} \pm}$ because electroosmosis and streaming potential tend to zero at high salt concentrations(ref. 13).

If a membrane has no fixed charges and the solution does not contain electrolyte, $D^{\prime}$ and $D^{\prime \prime}$ disappear and we have $D=D^{\circ}$. If the membrane has fixed charges, the additional frictional interaction between fixed charges and water flow must be considered. The charge effect is predicted by $D^{\prime}$ of eq. (40). At the low concentration $D^{\prime}$ is large because $\tau 0$ increases with decreasing concentration of electrolyte, while at high concentrations $D^{\prime}$ tends to zero. On the other hand, $D^{\prime \prime}$ is zero at low electrolyte concentrations. Since the transport number of coions increases with increasing concentration of electrolyte, the absolute value of $D$ " would monotonically increase with concentration. 


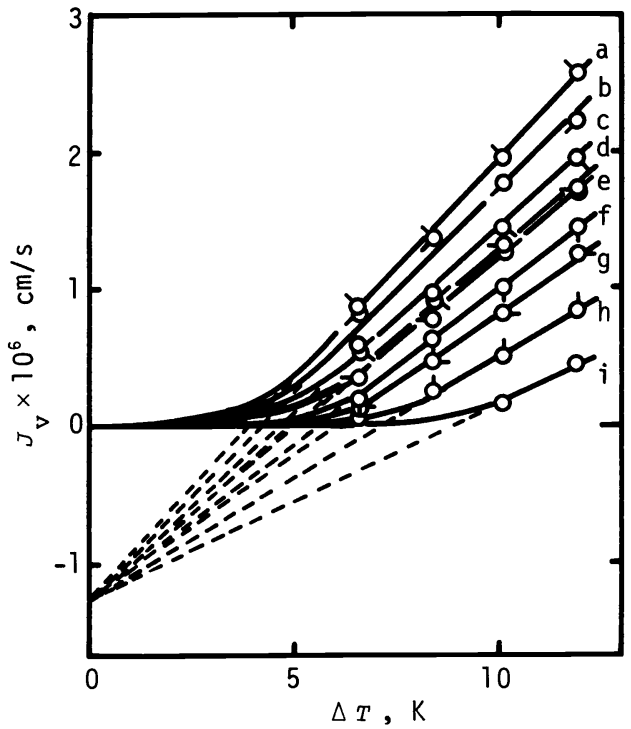

Fig. 11. The dependence of thermoosmotic flow on effective temperature difference with i$\mathrm{m}-2$ (ref. 5). KCl molalities (mol $/ \mathrm{kg}): a$, $0.001 ; b, 0.003 ; c, 0.01 ; d, 0.02 ; e, 0.05$; $\mathrm{f}, 0.1 ; \mathrm{g}, 0.2 ; \mathrm{h}, 0.5 ; \mathrm{i}, 0.93$.

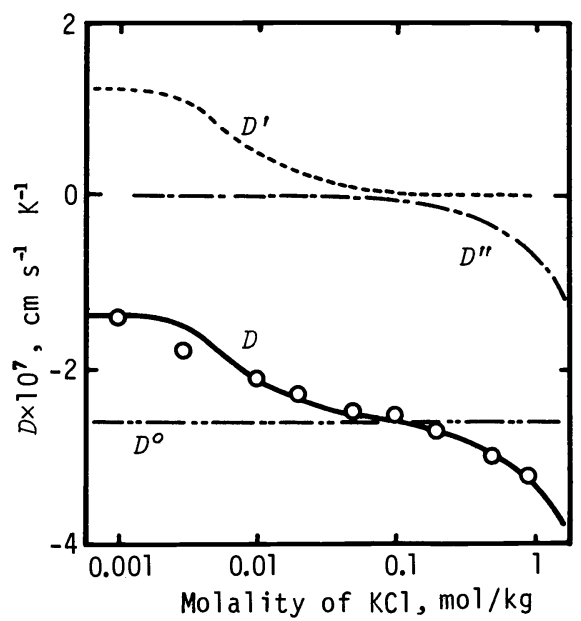

Fig. 12. The dependence of thermoosmotic coefficient, $D$, on $\mathrm{KCl}$ molality for membrane $i-m-2(r e f, 8)$.

The quantitative features of salt concentration dependence of $D^{\prime}$ and $D$ " are shown by broken lines in Fig. 12. Combination of three terms $D^{\circ}, D^{\prime}$, and $D^{\prime \prime}$ can qualitatively reproduce the experimental salt concentration dependence of $D$ in Fig. 12 .

For noncharged membranes $D^{\prime}=D^{\prime \prime}=0$ so that we have $D=D^{\circ}$. Since $D^{\circ} \propto\left(\overline{\bar{s}}_{0}-s_{0}\right)$ which is the difference between the transported entropy in membrane and the entropy in external solution, the direction of thermoosmosis may be sensitive to the membrane materials. In hydrophobic membranes we speculate that $\left(\overline{\bar{s}}_{0}-s_{0}\right)$ would be positive, so that the direction of thermoosmosis may be toward the cold side. In practice it was found that thermoosmosis occurred clearly toward the cold side when polytetrafluoroethylene membranes were used.

\section{Acknowledgement}

The author thanks Professor M. Nagasawa, Nagoya University, Japan for his continuing interest and helpful discussions.

\section{REFERENCES}

1. M. Tasaka, S. Morita, and M. Nagasawa, J. Phys. Chem., 69, 4191-4197 (1965).

2. M. Tasaka and M. Nagasawa, J. Polym. SCi. Symp., 49, 31-42 (1975).

3. M. Tasaka, K. Hanaoka, Y. Kurosawa, and C. Wada, Biophys. Chem., 3, 331-337 (1975).

4. M. Tasaka, N. Ichijo, S. Kobayashi, and H. Kobayashi, Biophys. Chem., 4, 269-274 (1976).

5. M. Tasaka, S. Abe, S. Sugiura, and M. Nagasawa, Biophys. Chem., 6, 271-278 (1977).

6. M. Tasaka, K. Ogawa, and T. Yamazaki, Biophys. Chem., 7, 279-283 (1978).

7. M. Tasaka, J. Membrane Sci., 4, 51-59 (1978).

8. M. Tasaka and M. Nagasawa, Biophys. Chem., 8, 111-116 (1978).

9. M. Tasaka, K. Kishi, and M. Okita, J. Membrane SCi., 17, 149-160 (1984).

10. M. Tasaka, Y. Kondo, and M. Nagasawa, J. Phys. Chem., 73, 3181-3188 (1969).

11. M. Tasaka and S. Nagasawa, Biophys. Chem., 4, 305-308 (1976).

12. M. Tasaka, C. Wada, and M. Nagasawa, J. Membrane SCi., 6, 171-183 (1980).

13. M. Tasaka, S. Tamura, N. Takemura, and K. Morimoto, J. Membrane SCi., 12, 169-182 (1982).

14. M. Tasaka, T. Okano, and T. Fujimoto, J. Membrane Sci., 19, 273-288 (1984).

15. M. Tasaka, S. Iwaoka, K. Yamagishi, Y. Ikeda, J. Membrane SCi., 24, 29-42 (1985).

16. T. Fujimoto, K. Ohkoshi, Y. Miyaki, and M. Nagasawa, Science, 224, 74-76 (1984).

17. M.S. Dariel and O. Kedem, J. Phys. Chem., 75, 336-342 (1975).

18. W.E. Goldstein and F.H. Verhoff, A. I. Ch. E. J., 21, 229-238 (1975).

19. F.S. Gaeta and D.G. Mita, J. Membrane Sci., 3, 191-214 (1978).

20. J.I. Mengual, J. Aguilar, and C. Fernandez-Pineda, J. Membrane SCi., 4, 209-219 (1978).

21. H. Vink and S.A.A. Chishti, J. Membrane SCi., I, 149-164 (1976).

22. R. Haase and C. Steinert, Z. Phys. Chem., N. F., 21, 270-297 (1959).

23. R.P. Rastogi, R.L. Blokha, and R.K. Agarwal, Trans. Faraday SoC., 60, 1386-1390 (1964). 
24. R. Haase and H.J. deGreiff, Z. Phys. Chem., N. F., 44, 301-313 (1965).

25. R. Haase and H.J. deGreiff, Z. Naturforsch., 26a, 1773-1774 (1971).

26. F.S. Gaeta and D.G. Mita, J. Phys. Chem., 83, 2276-2285 (1979).

27. D.G. Mita, F. Bellucci, M.G. Cutuli, and F.S. Gaeta, J. Phys. Chem., 86, 2975-2982 (1982).

28. F. Bellucci, E. Drioli, F.G. Summa, F.S. Gaeta, D.G. Mita, and N. Pagliuca, J. Chem. Soc., Faraday Trans. II, 75, 247-260 (1979).

29. L.G. Longsworth, J. Phys. Chem., 61, 1557-1562 (1957).

30. S.R. deGroot, Thermodynamics of Irreversible Processes, North-Holland Publishing Co., Amsterdam (1951)

31. S.R. deGroot and P. Mazur, Non-Equilibrium Thermodynamics, North-Holland Publishing Co., Amsterdam (1962).

32. O. Kedem and M.S. Dariel, Biological and Artificial Membranes and Desalination of Water, R. Passino, Ed., pp. 747-758, Elsevier Sci. Publishing Co., Amsterdam (1976).

33. J.G. Kirkwood, Ion Transport across Membranes, H.T. Clarke, Ed., p. 119, Academic Press, New York (1954).

34. H.J.V. Tyrrel, Diffusion and Heat Flow in Liquids, Butterworths, London (1961).

35. A.J. Staverman, Trans. Faraday Soc., 48, 176-185 (1952).

36. J.W. Lorimer, E.I. Boterenbrood, and J.J. Hermans, Discussions Faraday Soc., 21, 141149 (1956).

37. E. Riande, Physics of Electrolytes, Vol. 1, J. Hladik, Ed., chapt. Il, Academic Press, London-New York (1972).

38. N. Lakshminarayanaiah, Electrochemistry(Specialist Periodical Reports), Vol. 7, chapt. 2, The Chemical Society, London (1980).

39. N. Lakshminarayanaiah, Transport Phenomena in Membranes, chapt. 7, Academic Press, NewYork-London (1969).

40. G. Scibona, B. Scuppa, C. Fabiani, and C. Ciavola, Biochim. Biophys. Acta, 506, 30-41 (1978).

41. K.S. Pitzer and L. Brewer, Thermodynamics, 2nd Edition, chapts. 12 and 32, McGraw-Hill, New York (1961).

42. G. Scibona, B. Scuppa, C. Fabiani, and M. Pizzichini, Biochim. Biophys. Acta, 512, 41-53 (1978).

43. G. Scibona, C. Fabiani, B. Scuppa, and P.R. Danesi, Biophys. J., 16, 691-702 (1976).

44. G. Scibona, M. Magini, B. Scuppa, A. Castagnola, and C. Fabiani, Anal. Chem., 49, 212216 (1977).

45. C. Fabiani, B. Scuppa, G. Scibona, and M. Pizzichini, Gaz. Chim. Ital., 109, 409-414 (1979). 